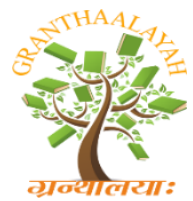

\author{
INTERNATIONAL JOURNAL OF RI
GRANTHAALAYAH \\ A knowledge Repository
}

Science

\title{
THE CORDIAL LABELING FOR THE CARTESIAN PRODUCT BETWEEN PATHS AND CYCLES
}

\author{
Mohammed M. Ali Al-Shamiri ${ }^{* 1,2}$, A. Elrokh ${ }^{3}$, Yasser El-Mashtawye ${ }^{1}$, S. Euat Tallah ${ }^{3}$ \\ ${ }^{1}$ Department of Mathematics, Faculty of Science and Arts, Mahayil Asir, King Khalid \\ University, K.S.A. \\ ${ }^{2}$ Department of Mathematics and Computer, Faculty of Science, Ibb University, Ibb, Yemen \\ ${ }^{3}$ Department of Mathematics, Faculty of Science, Menoufia University, Shebeen Elkom, Egypt
}

\begin{abstract}
A graph is said to be cordial if it has a 0-1 labeling that satisfies certain properties. In this paper we show the Cartesian product of a path and a cycle or vice versa are always cordial under some conditions. Also, we prove that the Cartesian product of two paths is cordial.

Keywords: Cartesian Product; Cordial; Cycle; Graph; Labeling; Path.

Cite This Article: Mohammed M. Ali Al-Shamiri, A. Elrokh, Yasser El -Mashtawye, and S. Euat Tallah. (2020). "THE CORDIAL LABELING FOR THE CARTESIAN PRODUCT BETWEEN PATHS AND CYCLES." International Journal of Research - Granthaalayah, 8(3), 331-341. https://doi.org/10.29121/granthaalayah.v8.i3.2020.166.
\end{abstract}

\section{Introduction}

It is known that graph theory and its branches have become interest topics for almost all fields of mathematics and also other area of science such as chemistry, biology, physics, communication, economics, and computer science. A graph labeling is an assignment of integers to the vertices or edges, or both, subject to certain conditions. There are many contributions and different kinds of labeling [6-10,11]. Suppose that $G=(V, E)$ is a graph, where V is the set of its vertices and $\mathrm{E}$ is the set of its edges Throughout, it is assumed $\mathrm{G}$ is connected, finite, simple and undirected. A binary vertex labeling of $\mathrm{G}$ is a mapping $f: V \rightarrow\{0,1\}$ in which $f(u)$ is said to be the labeling of $u \in V$.For an edge $e=u v \in E$, where $u, v \in V$, the introduced edge labeling $f^{*}: E \rightarrow\{0,1\}$ is defined by the formula $f^{*}(u w)=|f(v)+f(w)|(\bmod 2)$.Thus,for any edge e, $f^{*}(e)=0$ if its two vertices have the same labeling and $f^{*}(e)=1$ if they have different labeling.Let us denote $V_{0}$ and $V_{1}$ be the numbers of vertices labeled by 0 and 1 in $V$ respectively,and let $E_{0}$ and $E_{1}$ be the corresponding numbers of edges in $E$ labeled by 0 and 1 respectively. A binary vertex labeling $f$ of $\mathrm{G}$ is said to be cordial if $\left|V_{0}-V_{1}\right| \leq 1$ and $\left|E_{0}-E_{1}\right| \leq 1$.A graph $G$ is cordial if it has cordial labeling. The cordial graphs were introduced by Cahit [1] as a weaker version of both graceful and harmonious graphs [7-9]. A lot of operations on graphs have been applied on the cordial 
labeling[ 2-5].The Cartesian product of two graphs is that Boolean operation $G=G_{1} \times G_{2}$ in which for any two points $u=\left(u_{1}, u_{2}\right)$ and $v=\left(v_{1}, v_{2}\right)$, the line $u v$ is in $X$ whenever $\left[u_{1}=\right.$ $v_{1}$ and $\left.u_{2} v_{2} \in X_{2}\right]$ or $\left[u_{2}=v_{2}\right.$ and $u_{1} v_{1} \in X_{1}$ ]where $X, X_{1}, X_{2}$ are the sets of edges of $G$, $G_{1}$ and $G_{2}$ respectively [12]. It follows from the definition of the Cartesian product that the graph $\left.G_{1} \times G_{2}\right)$ has $n_{1} . n_{2}$ vertices and $n_{1} m_{2}+n_{2} m_{1}$ edges where $G_{1}$ has

$n_{1}, m_{1}$ vertices and edges and $G_{2}$ has $n_{2}, m_{2}$ vertices and edges. In this paper we show that $P_{n} \times C_{m}, C_{n} \times P_{m}$ and $P_{n} \times P_{m}$ are cordial for somen and $m$.

\section{Terminologies and Notation}

A path with $n$ vertices and $n-1$ edges is denoted by $P_{n}$, and a cycle with $n$ vertices and $n$ edges is denoted by $C_{n}$. Given a path or a cycle with $4 r$ vertices, we let $L_{4 r}$ denote the labeling $00110011 \ldots 0011$ (repeated $r$-times). In most cases, we modify this by adding symbols at one end or the other (or both ); thus $010 L_{4 r}$ denotes the labeling $01000110011 \ldots 0011$ of the path $P_{4 r+3}$ (or the cycle $C_{4 r+3}$ )when $r \geq 1$ and 010 when $r=0$, and so on [3-6]. We let $O_{r}$ denote the labeling $00 \ldots 0$ (zero repeated $r$ times), $1_{r}$ denote the labeling $11 \ldots 1$ (one repeated $r$ times) ,$M_{r}$ denotes to the labeling 0101...01if $\mathrm{r}$ is even and $0101 \ldots 010$ if $r$ is odd . The Cartesian product of two graphs is that Boolean operation $G=G_{1} \times G_{2}$ ) in which for any two points $u=\left(u_{1}, u_{2}\right)$ and $v=\left(v_{1}, v_{2}\right)$, the edgeuv is in $X$ whenever $\left[u_{1}=v_{1}\right.$ and $\left.u_{2} v_{2} \in X_{2}\right]$ or $\left[u_{2}=\right.$ $v_{2}$ and $u_{1} v_{1} \in X_{1}$ ] where $X, X_{1}, X_{2}$ are the sets of edges of $G, G_{1}$ and $G_{2}$ respectively [12].See Figure 2.1. It follows from the definition of the Cartesian product that the graph $G_{1} \times G_{2}$ has $n_{1} n_{2}$ vertices and $n_{1} m_{2}+n_{2} m_{1}$ edges, where $G_{1}$ has $n_{1}, m_{1}$ vertices and edges, $G_{2}$ has $n_{2}, m_{2}$ vertices and edges.It is easy to see that $G_{1} \times G_{2}$ is not isomorphic to $G_{2} \times G_{1}$.

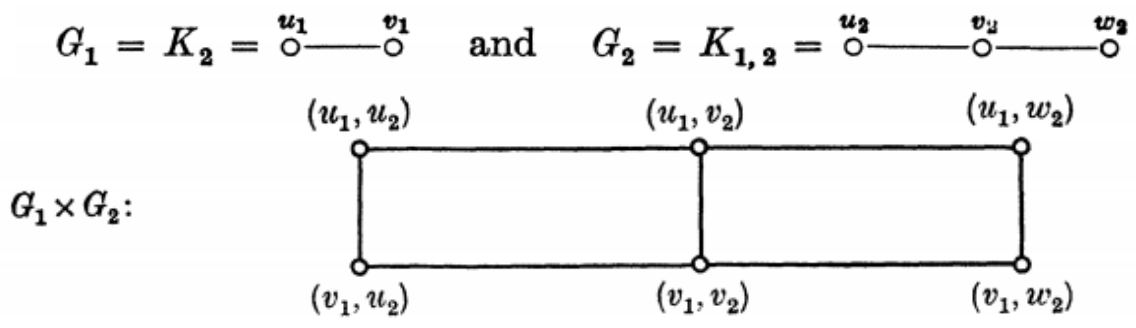

Figure 2.1.

\section{The Cordiality of The Product of a Path and a Cycle}

In this section, we show that the Cartesian product graphs $P_{n} \times C_{m}$ is cordial for some $\mathrm{n}$.

Lemma 3.1: The graph $P_{n} \times C_{n}$ is cordial if $\mathrm{n}$ is an even number.

Proof: We shall study two cases for $n \equiv i(\bmod 4)$ and $i=0,2$. Suppose that $n=2 \mathrm{~s}$

Case (1): At $i=0$ we choose the following labeling:

The $\left[1^{s t}, 3^{r d}, 5^{\text {th }}, \ldots,(2 \mathrm{~s}-3)^{\text {th }}\right]$ rows are labeled by $O_{2 s}$.

The $\left[2^{\text {nd }}, 4^{\text {th }}, 6^{\text {th }}, \ldots,(2 \mathrm{~s}-2)^{\text {th }}\right]$ rows are labeled by $1_{2 s}$.

The $(2 s-1)^{t h}$ row is labeled by $M_{2 s}$. 
The $(2 s)^{t h}$ row is labeled by $L_{2 s}$.In this case:

$V_{0}=V_{1}=[(2 s)(s-1)]+[s+s]=2 s^{2}$,

$E_{0}=$ the vertical edges plus the horizontal edges $=[s+s]+[2 s(2 s-2)+(s)]=s(4 s-1)$

$E_{1}=$ the vertical edges plus the horizontal edges $=[(2 s)(2 s-3)+s+(s)]+[2 s+s]=$ $s(4 s-1)$.Therefore $\left|E_{0}-E_{1}\right|=0$ and $\left|v_{1}-v_{1}\right|=0$ which satisfies the cordiality conditions

Case (2): Fori $=2$ we can apply the following labeling:

The $\left[1^{s t}, 3^{r d}, 5^{\text {th }}, \ldots,(2 \mathrm{~s}-3)^{\text {th }}\right]$ rows are labeled by $O_{2 s}$.

The $\left[2^{\text {nd }}, 4^{\text {th }}, 6^{\text {th }}, \ldots,(2 \mathrm{~s}-2)^{\text {th }}\right]$ rows are labeled by $1_{2 s}$.

The $(2 s-1)^{t h}$ row is labeled by $M_{2 s}$.

The $(2 s)^{\text {th }}$ row is labeled by $L_{2 s-2} 10$.In this situation:

$V_{0}=V_{1}=[(2 s)(s-1)]+[s+s]=2 s^{2}$,

$E_{0}=$ thehorizontal edges plus thevertical edges $=[2 s(2 s-2)+(s+1)]+[(s-1)+s]=$ $s(4 s-1)$

$E_{1}=$ thehorizontal edges plus thevertical edges $=[2 s+(s-1)]+[(2 s)(2 s-3)+s+(s+$ $1)]=s(4 s-1)$ Therefore, $\left|E_{0}-E_{1}\right|=0,\left|v_{1}-v_{1}\right|=0$ so $P_{n} \times C_{n}$ where $n$ is even is cordial as we wished to show. The following examples demonstrate some examples for the previous cases:

$$
P_{4} \times C_{4}(\text { for } s=2) v_{0}=v_{1}=8, E_{0}=E_{1}=14 \text { i.e }\left|E_{0}-E_{1}\right|=0,\left|v_{1}-v_{1}\right|=0 \text {. }
$$

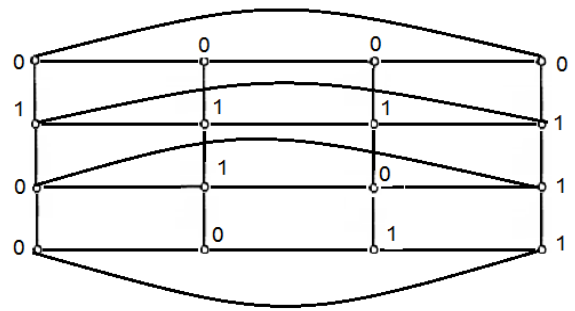

Figure 3.1.

$P_{6} \times C_{6}($ for $s=3)$

$$
v_{0}=v_{1}=18, E_{0}=E_{1}=33 \text {, i.e. }\left|E_{0}-E_{1}\right|=0,\left|v_{0}-v_{1}\right|=0 \text {. }
$$

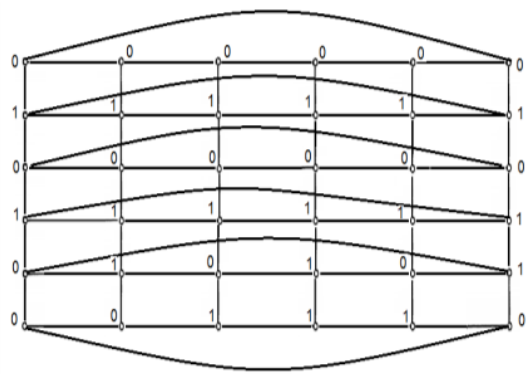

Figure 3.2.

Lemma 3.2: The graph $P_{n} \times C_{m}$ is cordial for all $\mathrm{n}$ is even and $\mathrm{m}$ is odd such that $n-m=-1$ also it is cordial for all $\mathrm{n}$ is odd and $\mathrm{m}$ is even which satisfy $n-m=1$.

Proof: We divide the proof into two cases: 
Case (1): Suppose that $n=2 s$ and $m=2 s+1$ that satisfy the previous condition i.e. $n-m=$ -1 . Then we can use the following labeling:

The $\left[1^{s t}, 3^{r d}, 5^{t h}, \ldots,(2 \mathrm{~s}-3)^{\text {th }}\right]$ rows are labeled by $O_{2 s+1}$.

The $\left[2^{\text {nd }}, 4^{\text {th }}, 6^{\text {th }}, \ldots,(2 s-2)^{\text {th }}\right]$ rows are labeled by $1_{2 s+1}$.

The $(2 s-1)^{t h}$ row is labeled by $M_{2 r+1}$.

The $(2 s)^{\text {th }}$ row is labeled by $M_{2 r} 1$. Then we will get : $v_{0}=v_{1}=[(2 s+1)(s-1)]+[s+1+$ $s]=s(2 s+1)$,

$E_{0}=$ the vertical edges plus the horizontal edges $=[\mathrm{s}+2 \mathrm{~s}]+[(2 s+1)(2 s-2)+(1+1)]=$ $s(4 s+1)$

$E_{1}=$ the vertical edges plus the horizontal edges $=[s(2 s-3)+(2 s-1)+s(2 s-2)]=$ $s(4 s+1)-1$.

i.e. $\left|E_{0}-E_{1}\right|=1,\left|v_{0}-v_{1}\right|=0$ so $P_{2 s} \times C_{2 s+1}$ is cordial as we wanted to show For example: (for $s=2) P_{4} \times C_{5}$

$v_{0}=v_{1}=10, E_{0}=18, E_{1}=17$,i.e. $\left|E_{0}-E_{1}\right|=1,\left|v_{0}-v_{1}\right|=0$

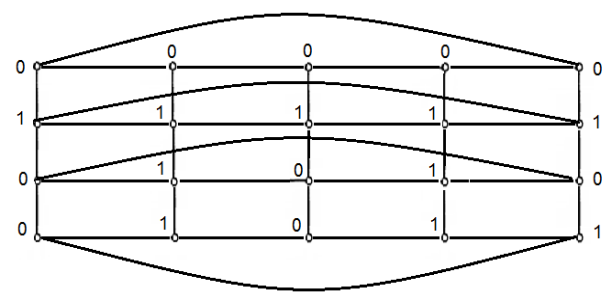

Figure 3.3.

Case (2): Suppose that $n=2 s+1$ and $m=2 s$.It is obvious that $n-m=1$.

By using the following labeling:

The $\left[1^{s t}, 3^{r d}, 5^{t h}, \ldots,(2 s-1)^{\text {th }}\right]$ rows are labeled by $O_{2 s}$.

The $\left[2^{\text {nd }}, 4^{\text {th }}, 6^{\text {th }}, \ldots,(2 \mathrm{~s})^{\text {th }}\right]$ rows are labeled by $1_{2 s}$.

The $\left[(2 s+1)^{t h}\right.$ row is labeled by $M_{2 s}$. Therefore,

$$
v_{0}=v_{1}=[(2 s)(s)]+[s]=s(2 s+1),
$$

$E_{0}=$ the vertical edges plus the horizontal edges $=[s]+[(2 s)(2 s)]=s(4 s+1)$.

$E_{1}=$ the vertical edges plus the horizontal edges $=[s(2 s)+s(2 s-1)]+[2 s]=s(4 s+1)$.

i.e. $\left|E_{0}-E_{1}\right|=1,\left|v_{0}-v_{1}\right|=0 \quad$ so $P_{2 s+1} \times C_{2 s}$ is cordial as we wanted to show.

For example,: (for $s=2$ ) $P_{5} \times C_{4}$

$v_{0}=v_{1}=10, E_{0}=E_{1}=18,\left|E_{0}-E_{1}\right|=1,\left|v_{0}-v_{1}\right|=0$.

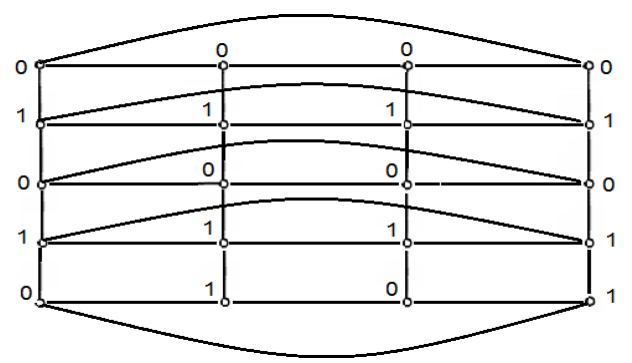

Figure 3.4. 
Lemma 3.3: The graph $P_{n} \times C_{n}$ is cordial if $n$ is an odd number.

Proof: Suppose that $n=2 s+1$.Then one can take the following labeling:

The $\left[1^{s t}, 3^{r d}, 5^{\text {th }}, \ldots,(2 s-1)^{\text {th }}\right]$ rows are labeled by $O_{2 s+1}$.

The $\left[2^{\text {nd }}, 4^{\text {th }}, 6^{\text {th }}, \ldots,(2 \mathrm{~s})^{\text {th }}\right]$ rows are labeled by $1_{2 s+1}$.

The $(2 s+1)^{\text {th }}$ row is labeled by $M_{2 s+1}$. Therefore,

$v_{0}=[(2 s+1)(s)]+[s+1]=2 s(s+1)+1, v 1=[(2 s+1)(s)]+[s]=2 s(s+1)$,

$E_{0}=$ the vertical edges plus the horizontal edges $=[s]+[2 s(2 s+1)+1]=s(4 s+3)+1$.

$E_{1}=$ the vertical edges plus the horizontal edges $=[(s+1)+(2 s+1)(2 s-1)]+[2 s]=$ $s(4 s+3)$.

i.e. $\left|E_{0}-E_{1}\right|=1,\left|v_{0}-v_{1}\right|=1$ so $P_{2 s+1} \times C_{2 s+1}$ is cordial as we wanted to prove.

For example: (for $\mathrm{s}=2) \quad P_{5} \times C_{5}$

$v_{0}=13, v_{1}=12, \quad E_{0}=23, \quad E_{1}=22$ i.e. $\left|E_{0}-E_{1}\right|=1, \quad\left|v_{0}-v_{1}\right|=1$.

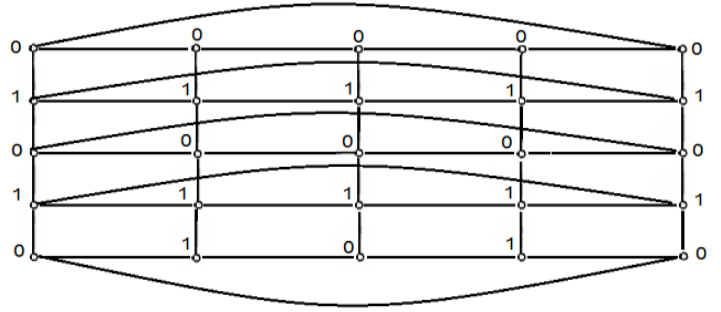

Figure 3.5.

Now, from the previous lemmas we have the following conclusion:

Theorem 3.1: The Cartesian product graph $P_{2 s+i} \times C_{2 s+j}$ is cordial for all $\mathrm{i}$ and $\mathrm{j}$ where $(i, j=$ $0,1)$.

\section{The Cartesian Product of Two Paths}

In this section, we study the cordiality of Cartesian product between two paths.

Lemma 4.1: The graph $P_{n} \times P_{n}$ is cordial if $n$ is an even number.

Proof: Suppose that $n=2 s$, then we choose the following labeling:

The $\left[1^{s t}, 3^{r d}, 5^{t h}, \ldots,(2 s-1)^{\text {th }}\right]$ rows are labeled by $O_{2 s}$.

The $\left[2^{\text {nd }}, 4^{\text {th }}, 6^{\text {th }}, \ldots,(2 \mathrm{~s})^{\text {th }}\right]$ rows are labeled by $1_{2 s}$. Then we get : $v_{0}=v_{1}=s(2 s)$,

$E_{0}=$ the vertical edges plus the horizontal edges $=0+[2 s(2 s-1)]=2 s(2 s-1)$

$E_{1}=$ the vertical edges plus the horizontal edges $=[2 s(2 s-1)]+0=2 s(2 s-1)$

i.e. . $\left|E_{0}-E_{1}\right|=0,\left|v_{0}-v_{1}\right|=0$. So, $P_{2 s} \times P_{2 s}$ is cordial as we wanted to show.

For example, $P_{4} \times P_{4}$ :

$$
v_{0}=v_{1}=8, E_{0}=E_{1}=12 \text {, i.e. }\left|E_{0}-E_{1}\right|=0,\left|v_{0}-v_{1}\right|=0 .
$$






Figure 4.1.

Lemma 4.2: The graph $P_{n} \times P_{m}$ is cordial for all $n$, where $n$ is even and $m$ is odd such that $n-$ $m=-1$ also it is cordial for all $n$ is odd and $m$ is even such that $n-m=1$.

Proof: We divide the proof into two cases:

Case (1): Suppose that $n=2 s$ and $m=2 s+1$ which satisfy the previous condition $n-m=$ -1 .Therefore, we can use the following labeling:

The $\left[1^{s t}, 3^{r d}, 5^{t h}, \ldots,(2 s-1)^{\text {th }}\right]$ rows are labeled by $O_{2 s+1}$.

The $\left[2^{\text {nd }}, 4^{\text {th }}, 6^{\text {th }}, \ldots,(2 \mathrm{~s})^{\text {th }}\right]$ rows are labeled by $1_{2 s+1}$.In this case :

$v_{0}=v_{1}=s(2 s+1)$,

$E_{0}=$ the vertical edges plus the horizontal edges $=[0]+[2 s(2 s)]=4 s^{2}$,

$E_{1}=$ the vertical edges plus the horizontal edges $=[(2 s+1)(2 s-1)]+0=(2 s+1)(2 s-1)$ , i.e. $\left|E_{0}-E_{1}\right|=1,\left|v_{0}-v_{1}\right|=0$ so $P_{2 s} \times P_{2 s+1}$ is cordial as we wanted to show

For example $P_{4} \times P_{5}$ :

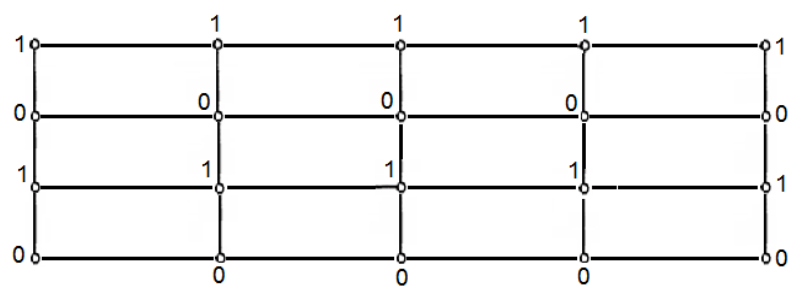

Figure 4.2.

Case (2): Suppose that $n=2 s+1$ and $m=2 s$.It is obvious that $n-m=1$. Therefore, we use the following labeling:

The $\left[1^{s t}, 3^{r d}, 5^{\text {th }}, \ldots,(2 s-1)^{\text {th }}\right]$ columns are labeled by $O_{2 s+1}$.

The $\left[2^{\text {nd }}, 4^{\text {th }}, 6^{\text {th }}, \ldots,(2 \mathrm{~s})^{\text {th }}\right]$ columns are labeled by $1_{2 s+1}$. In this case:

$v_{0}=v_{1}=s(2 s+1)$,

$E_{0}=$ the vertical edges plus the horizontal edges $=[2 s(2 s)]+0=\left(4 s^{2}\right)$,

$E_{1}=$ the vertical edges plus the horizontal edges $=0+[(2 s+1)(2 s-1)]=$

$(2 s+1)(2 s-1)$,i.e. $\left|E_{0}-E_{1}\right|=1,\left|v_{0}-v_{1}\right|=0$ so $P_{2 s+1} \times P_{2 s}$ is cordial as we wanted to show .

For example $P_{5} \times P_{4}$ :

$$
v_{0}=v_{1}=10, \quad E_{0}=16, E_{1}=15 \text {, i.e. }\left|E_{0}-E_{1}\right|=1, \quad\left|v_{0}-v_{1}\right|=0
$$




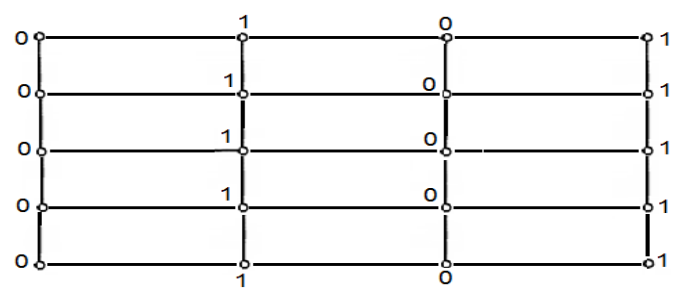

Figure 4.3.

Lemma 4.3: The graph $P_{n} \times P_{n}$ is cordial for all odd numbers $n$.

Proof: Suppose that $n=2 s+1$. Therefore, one we take the following labeling: The $\left[1^{\text {st }}, 2^{\text {nd }}\right]$ columns are labeled by the sequence $\left[1_{s} 0_{s+1}\right]$.

The $\left[3^{\text {rd }}, 5^{\text {th }}, \ldots,(2 s+1)^{\text {th }}\right]$ columns are labeled by the sequence $\left[0{ }_{s} 1_{s+1}\right]$.

The $\left[4^{\text {th }}, 6^{\text {th }}, \ldots,(2 \mathrm{~s})^{\text {th }}\right]$ columns arelabeled by the sequence $\left[1_{s} 0_{s+1}\right]$. Then:

$v_{0}=(s+1)(s+1)+s(s)=2 s(s+1)+1, v_{1}=s(s+1)+s(s+1)=2 s(2 s+1)$, $E_{0}=$ the vertical edges plus the horizontal edges $=[(2 s-1)(s+2)+(2 s-1)(s-1)]+$ $[(2 s+1)]=2 s(2 s+1)$,

$E_{1}=$ the vertical edges plus the horizontal edges $=[(2 s+1)]+[(2 s-1)(2 s+1)]=2 s(2 s+$ $1)$, i.e. $\left|E_{0}-E_{1}\right|=0,\left|v_{0}-v_{1}\right|=1$.So $P_{2 s+1} \times P_{2 s+1}$ is cordial as we wanted to show .

For example $P_{5} \times P_{5}$ :

$v_{0}=13, v_{1}=12, E_{0}=E_{1}=20$, i.e. $\left|E_{0}-E_{1}\right|=0,\left|v_{0}-v_{1}\right|=1$.

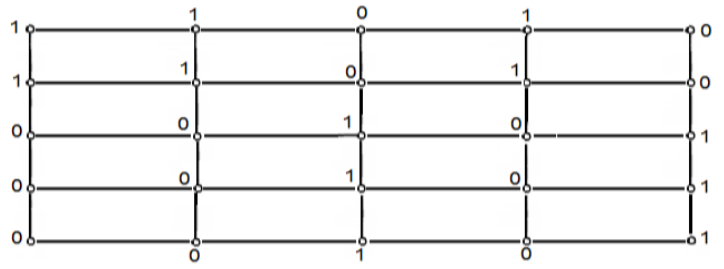

Figure 4.4.

The prove of the following theorem comes directly from the previous lemmas

Theorem4.1: The Cartesian product graph $P_{2 s+i} \times P_{2 s+j}$ is cordial for all $i$ and $j$ where $(i, j=$ $0,1)$.

\section{The Cartesian Product of a Cycle and A Path}

In this section, we will study the cordiality of a cycle and a path.

Lemma 5.1: The graph $C_{n} \times P_{n}$ is cordial if $n$ is an even number.

Proof: Suppose that $n=2 s$. Therefore, we study two cases for $n \equiv i(\bmod 4)$ and $i=0,2$.

Case (1): At $i=0$ we choose the following labeling:

The $\left[1^{s t}, 3^{r d}, 5^{\text {th }}, \ldots,(2 \mathrm{~s}-3)^{\text {th }}\right]$ columns are labeled by $O_{2 s}$. 
The $\left[2^{\text {nd }}, 4^{\text {th }}, 6^{\text {th }}, \ldots,(2 s-2)^{\text {th }}\right]$ columns are labeled by $1_{2 s}$.

The $(2 s-1)^{t h}$ column is labeled by $M_{2 s}$. The $(2 s)^{t h}$ column is labeled by $L_{2 s}$. Then:

$V_{0}=V_{1}=[(2 s)(s-1)]+[s+s]=2 s^{2}$,

$E_{0}=$ the vertical edges plus the horizontal edges $=[2 s(2 s-2)+(s)]+[s+s]=s(4 s-1)$

$E_{1}=$ the vertical edges plus the horizontal edges $=[2 s+s]+[(2 s)(2 s-3)+s+(s)]=$ $s(4 s-1)$ Then: $\left|E_{0}-E_{1}\right|=0,\left|v_{0}-v_{1}\right|=0$ which satisfies the cordiality conditions.

For example: $C_{4} \times P_{4}($ for $s=2)$

$$
v_{0}=v_{1}=8, E_{0}=E_{1}=14 \text { i.e }\left|E_{0}-E_{1}\right|=0,\left|v_{1}-v_{1}\right|=0 \text {. }
$$

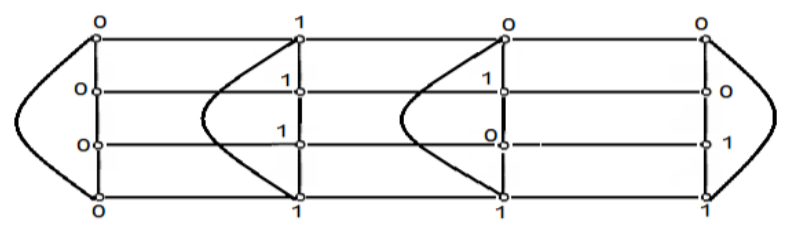

Figure 5.1.

Case (2): For $i=2$ we can apply the following labeling:

The $\left[1^{s t}, 3^{r d}, 5^{t h}, \ldots,(2 \mathrm{~s}-3)^{\text {th }}\right]$ columns are labeled by $O_{2 s}$.

The $\left[2^{\text {nd }}, 4^{\text {th }}, 6^{\text {th }}, \ldots,(2 \mathrm{~s}-2)^{\text {th }}\right]$ columns are labeled by $1_{2 s}$.

The $(2 s-1)^{t h}$ column is labeled by $M_{2 s}$.

The $(2 s)^{\text {th }}$ column is labeled by $L_{2 s-2} 10$.In this case:

$V_{0}=V_{1}=[(2 s)(s-1)]+[s+(s-1)+1]=2 s^{2}$,

$E_{0}=$ the vertical edges plus the horizontal edges $=[2 s(2 s-2)+0+(s+1)]+[(s-1)+$ $s]=s(4 s-1)$

$E_{1}=$ the vertical edges plus the horizontal edges $=[2 s+(s-1)]+[(2 s)(2 s-3)+s+(s+$ $1)]=s(4 s-1)$.Then: $\left|E_{0}-E_{1}\right|=0,\left|v_{0}-v_{1}\right|=0$ which is cordial.

For example: $C_{6} \times P_{6}$

$v_{0}=v_{1}=18, E_{0}=E_{1}=33$, i.e. $\left|E_{0}-E_{1}\right|=0,\left|v_{0}-v_{1}\right|=0$.

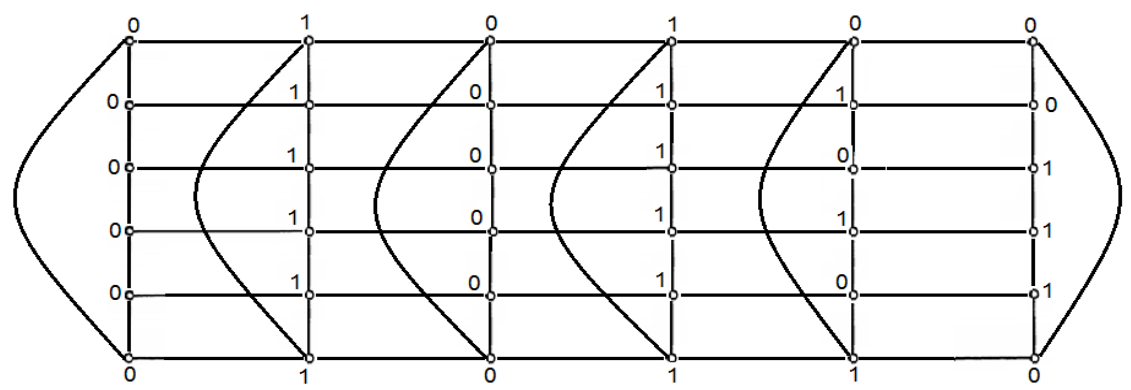

Figure 5.2 
Lemma 5.2: The graph $C_{n} \times P_{m}$ is cordial for all $n$ is even and $m$ is odd such that $n-m=-1$ also it is cordial for all $n$ is odd and $m$ is even which satisfy $n-m=1$.

Proof: we divide the proof into two cases:

Case (1): Suppose that $n=2 s$ and $m=2 s+1$ which satisfy the previous condition $n-m=-1$. we can use the following labeling:

The $\left[1^{s t}, 3^{r d}, 5^{\text {th }}, \ldots,(2 \mathrm{~s}-1)^{\text {th }}\right]$ columns are labeled by $O_{2 s}$.

The $\left[2^{\text {nd }}, 4^{\text {th }}, 6^{\text {th }}, \ldots,(2 \mathrm{~s})^{\text {th }}\right]$ columns are labeled by $1_{2 s}$.

The $(2 s+1)^{t h}$ column is labeled by $M_{2 s}$.In this case

$V_{0}=V_{1}=(s)(2 s)+s=s(2 s+1)$,

$E_{0}=$ the vertical edges plus the horizontal edges $=[2 s(2 s)+(s)]=s(4 s+1)$

$E_{1}=$ the vertical edges plus the horizontal edges $=[2 s]+[(2 s)(s)+(2 s-1)(s)]=s(4 s+$ 1)

Then: $\left|E_{0}-E_{1}\right|=0,\left|v_{0}-v_{1}\right|=0$.So $C_{2 s} \times P_{2 s+1}$ is cordial as we wished.

For example: $C_{4} \times P_{5}$

$$
v_{0}=v_{1}=10, E_{0}=E_{1}=18 \text { i.e. }\left|E_{0}-E_{1}\right|=0,\left|v_{0}-v_{1}\right|=0 \text {. }
$$

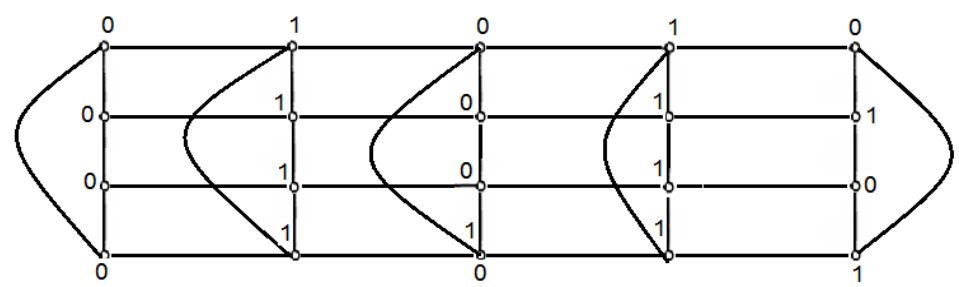

Figure 5.3

Case (2): Suppose that $n=2 s+1$ and $m=2 s$.It is obvious that $n-m=1$.

We use the following labeling:

The $\left[1^{s t}, 3^{r d}, 5^{t h}, \ldots,(2 \mathrm{~s}-3)^{\text {th }}\right]$ columns are labeled by $O_{2 s+1}$.

The $\left[2^{\text {nd }}, 4^{\text {th }}, 6^{\text {th }}, \ldots,(2 \mathrm{~s}-2)^{\mathrm{th}}\right]$ column are labeled by $1_{2 s+1}$.

The $(2 s-1)^{t h}$ column is labeled by $M_{2 s+1}$.

The $(2 s)^{t h}$ column is labeled by $M_{2 s} 1$. In this situation:

$$
v_{0}=v_{1}=(2 s+1)(s-1)+s+(s+1)=s(2 s+1) \text {, }
$$

$E_{0}=$ the vertical edges plus the horizontal edges $=[(2 s+1)(2 s-2)+1+1]+[0+s+$ $2 s]=s(4 s+1)$,

$E_{1}=$ the vertical edges plus the horizontal edges $=[2 s+2 s]+[s(2 s-3)+s(2 s-2)+$ $(2 s-1)]=s(4 s+1)-1$. Then $\left|E_{0}-E_{1}\right|=1,\left|v_{0}-v_{1}\right|=0$ which satisfy cordiality conditions.

For example: $C_{5} \times P_{4}$

$$
v_{0}=v_{1}=10, E_{0}=18, E_{1}=17 \text { then }\left|E_{0}-E_{1}\right|=0,\left|v_{0}-v_{1}\right|=0 \text {. }
$$




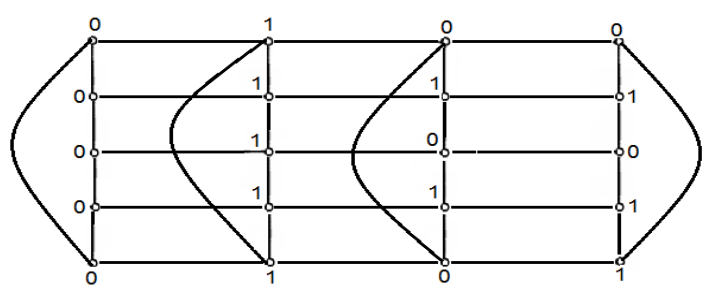

Figure 5.4.

Lemma 5.3. The graph $C_{n} \times P_{n}$ is cordial for all $n$ is an odd number.

Proof: Suppose that $n=2 s+1$ we take the following labeling:

The $\left[1^{s t}, 3^{r d}, 5^{t h}, \ldots,(2 s-1)^{\text {th }}\right]$ columns are labeled by $O_{2 s+1}$.

The $\left[2^{\text {nd }}, 4^{\text {th }}, 6^{\text {th }}, \ldots,(2 \mathrm{~s})^{\text {th }}\right]$ columns are labeled by $1_{2 s+1}$.

The $(2 s+1)^{t h}$ column is labeled by $M_{2 s+1}$.

Then: $v_{0}=(2 s+1)(s)+(s+1)=2 s(s+1)+1, v_{1}=(2 s+1)(s)+s=2 s(s+1)$,

$E_{0}=$ the vertical edges plus the horizontal edges $=[(2 s+1)(2 s)+1]+[s]=s(4 s+3)+1$, $E_{1}=$ the vertical edges plus the horizontal edges $=[2 s]+[(2 s-1)(2 s+1)+(s+1)]=$ $s(4 s+3)$ then $\left|E_{0}-E_{1}\right|=1,\left|v_{1}-v_{1}\right|=1$. So $C_{2 s+1} \times P_{2 s+1}$ is cordial as we wanted to show.

For example: $C_{5} \times P_{5}$

$v_{0}=13, v_{1}=12, E_{0}=23, E_{1}=22$ then $\left|E_{0}-E_{1}\right|=1,\left|v_{0}-v_{1}\right|=1$.

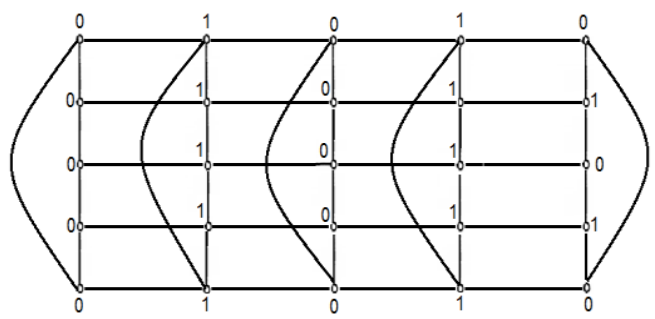

Figure 5.5.

As a direct consequence of the last three lemmas we have

Theorem 5.1: The Cartesian product graph $C_{2 s+i} \times P_{2 s+j}$ is cordial for alli and $\mathrm{j}$ where $(\mathrm{i}, \mathrm{j}=$ $0,1)$.

\section{References}

[1] I.Cahit, On cordial and 3-equitable labeling of graphs,Utilities Math.,37(1990).

[2] A. T. Diab, Study of some problems of cordial graphs, ARS Combinatoria. 2009; 92:255-261.

[3] A.T.Diab, On Cordial Labeling of the second power of paths with other graphs, ARS Combinatoria 2010; 97A:327-343.

[4] A. T.Diab, Generalization of some results on cordial graphs , ARS Combinatoria. 2009; 92:161173.

[5] A.T.Diab,On Cordial Labeling of Wheels with other graphs ,ARS Combinatoria 2011;100:265279. 
[6] J.A.Gallian,A dynamic survey of graph labeling, The electronic Journal of Combinatorics 17,November 2010:13 DS6.

[7] S. W.Golomb, How to number a graph in graph theory and Computing, R.C. Read,rd., Academic Press,New York. 1972: 23-37.

[8] R.L.Graham and N.J.A.Sloane, On additive bases and harmonious graphs, SIAM J. Alg. Discrete Math.1. 1980:382-404.

[9] A.Rosa,On certain valuations of the vertices of a graph,Theorey of graphs (Internet Symposium , Rome,July 1966),Gordon and Breach,N.Y. and Dunod Paris. 1967:349-355.

[10] M.A Seoud and Abdel Maqusoud, On Cordial and balanced labeling af graphs,J. Egypt. Math. Soc. 1999; 7:127-135.

[11] Mohammed M. Ali Al-Shamiri, Shokry I. N, A. I. Elrokh, Yasser Elmshtaye, Some Results on Cordial Digraphs, Open Journal of Discrete Mathematics,2020;10;1:4-12.

[12] F. Harary and G.W.Wilcox, Boolean operations on graphs ,Math.Scand. 1967; 20: 41-51

\footnotetext{
*Corresponding author.

E-mail address: mal-shamiri@kku.edu.sa
} 